\title{
Flux-Weakening Control Research for Interior Permanent Magnet Synchronous Motor in Electric Vehicle
}

\author{
Li Lin ${ }^{1, a}$, Yong-kuang Li ${ }^{2, b}$, Hai-yan Zhao ${ }^{3, c}$ and Shu-qi Shi ${ }^{4, d}$ \\ ${ }^{1}$ Shaoyang University, Shaoyang, Hunan Province, China \\ ${ }^{2}$ Shaoyang University, Shaoyang, Hunan Province, China \\ ${ }^{3}$ Shaoyang University, Shaoyang, Hunan Province, China \\ ${ }^{4}$ Shaoyang University, Shaoyang, Hunan Province, China

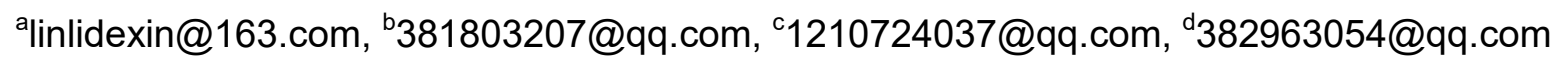

Keywords: electric traction;interior permanent magnet synchronous motor;Flux-Weakening

\begin{abstract}
In order to improve the vehicle speed range of the interior permanent magnet synchronous motor, the flux weakening control strategy is studied in this article. The interior permanent magnet synchronous motor field weakening control system simulation model is established based on the platform of MATLAB/Simulink, and high speed weak magnetic control system simulation is carried out. The simulation results show that the system has a wide range of speed regulation, and these provide a reference significance for further study on the high-speed cruise and overtaking and other conditions.
\end{abstract}

\section{Introduction}

Interior Permanent Magnet Synchronous Motor (IPMSM) due to its high torque power density, high power factor, wide constant power speed range advantages in electric vehicles, wind power, rail traction, electric spindle and other fields has been widely used ${ }^{[1-3]}$. Electric vehicle drive system can realize constant torque control at low speed, and can achieve wide constant power speed at high speed. In addition, in the high speed traction rail transportation, vigorously pursued the IPMSM system wide constant power speed, in order to achieve the speed upgrade, therefore, in electric traction, the interior permanent magnet synchronous motor for weak magnetic control, enhance the speed range of IPMSM, which has an important significance ${ }^{[4-5]}$. In this paper, the interior permanent magnet motor field weakening control strategy is studied; With the MATLAB/Simulink simulation platform, the simulation model of IPMSM weak magnetic control system for vehicle is built, the simulation results show that the system has a wide range of speed regulation, and provides some reference for the further research on high speed cruise and overtaking condition.

\section{d-q axis mathematical model of IPMSM}

In order to simplify the analysis, the rotor is assumed to have no damping winding, that is, the damping winding has no damping effect on the permanent magnet of the motor; the stator air gap distribution is uniform; the counter electromotive force waveform is sinusoidal; the core of the motor is neglected, and the eddy current and the magnetic hysteresis loss of the motor are neglected.

Under these assumptions, the mathematical model of the d-q axis of IPMSM is:

Voltage equation:

\footnotetext{
Li Lin (1972.10-), male, Professor, doctor, master's tutor, mainly engaged in power electronics and power transmission, new energy electric vehicles and wind power and other aspects of teaching and research work.

Fund project: Hunan provincial science and technology department key research and development project (project number: 2015GK3033); Study on the teaching reform project of Hunan Province in 2015 the ordinary higher school: "Research on rail traffic signal control and professional knowledge structure and curriculum system" (Hunan general textbook 2015 No. 291). In 2009 in Hunan Province Department of education science research project (project number: 09C884); Shaoyang city in 2015 the scientific project (gas hot water boiler control system key technology research and application); project supported by the science and technology project of Hunan province (No. 2015GK2033). Shaoyang University graduate student research and innovation project (project number: Cx2015SY018).
} 


$$
\left\{\begin{array}{l}
u_{d}=R_{s} i_{d}+L_{d} \frac{\mathrm{d} i_{d}}{\mathrm{~d} t}-\omega L_{q} i_{q} \\
u_{q}=R_{s} i_{q}+L_{d} \frac{\mathrm{d} i_{q}}{\mathrm{~d} t}+\omega L_{q} i_{q}+\omega \psi_{\mathrm{f}}
\end{array}\right.
$$

Torque equation:

$$
\boldsymbol{T}_{e}=p\left(\psi_{d} i_{q}-\psi_{q} \boldsymbol{i}_{d}\right)=p\left[\psi_{\mathrm{f}} \boldsymbol{i}_{q}+\left(\boldsymbol{L}_{d}-\boldsymbol{L}_{q}\right) \boldsymbol{i}_{d} \boldsymbol{i}_{q}\right]
$$

Motion equation:

$$
T_{e}=T_{\mathrm{L}}+\frac{J}{p} \cdot \frac{\mathrm{d} \omega}{\mathrm{d} t}+F \frac{\omega}{p}
$$

In the equation, $\boldsymbol{i}_{\boldsymbol{d}} 、 \boldsymbol{i}_{q}, \boldsymbol{u}_{\boldsymbol{d}} 、 \boldsymbol{u}_{q}, \psi_{d}, \psi_{q}, \boldsymbol{L}_{\boldsymbol{d}} 、 \boldsymbol{L}_{q}$ respectively is the d-q axis of the stator current, voltage, flux and inductance components; $\psi_{\mathrm{f}}$ is a flux linkage produced by permanent magnet; $\boldsymbol{R}_{\boldsymbol{s}}$ is the stator phase winding resistance; $\omega$ is the electric motor speed; $\boldsymbol{p}$ is the pole pairs; $\boldsymbol{T}_{e} 、 \boldsymbol{T}_{\boldsymbol{L}} 、 \boldsymbol{F} 、 \boldsymbol{J}$ respectively is motor electromagnetic torque, load torque, viscous friction coefficient and moment of inertia.

\section{IPMSM weak magnetic control strategy}

The rotor exciting magnetic field of IPMSM is produced by permanent magnet, by increasing stator current and direct axis armature reaction to weaken the motor air gap magnetic field, to achieve the "weak magnetic" effect, and the terminal voltage and stator current are restricted by the output of the inverter, in the $\mathrm{d}-\mathrm{q}$ axis coordinate system, the limit values of the current and voltage are assumed to be $\boldsymbol{i}_{\lim } 、 \boldsymbol{u}_{\lim }$, the following formula can be obtained:

$$
\begin{aligned}
& \boldsymbol{i}_{s}=\sqrt{\boldsymbol{i}_{\boldsymbol{d}}^{2}+\boldsymbol{i}_{q}^{2}} \leq \boldsymbol{i}_{\mathrm{lim}} \\
& \boldsymbol{u}_{s}=\sqrt{\boldsymbol{u}_{d}^{2}+\boldsymbol{u}_{q}^{2}} \leq \boldsymbol{u}_{\mathrm{lim}}
\end{aligned}
$$

Because of the high speed steady state operation of the motor, the resistance value is much less than the reactance value, so the voltage drop on the resistance is negligible, by formula (1), it can be deduced that the steady-state voltage equation is:

$$
\left\{\begin{array}{l}
\boldsymbol{u}_{d}=-\omega \boldsymbol{L}_{q} \boldsymbol{i}_{q} \\
\boldsymbol{u}_{q}=\omega \boldsymbol{L}_{\boldsymbol{d}} \boldsymbol{i}_{d}+\omega \psi_{\mathrm{f}}
\end{array}\right.
$$

The equation (6) into the equation (5), finishing the voltage limit of elliptic equation:

$$
\left(\boldsymbol{L}_{d} \boldsymbol{i}_{\boldsymbol{d}}+\psi_{\mathrm{f}}\right)^{2}+\left(\boldsymbol{L}_{q} \boldsymbol{i}_{q}\right)^{2} \leq\left(\frac{\boldsymbol{u}_{\mathrm{lim}}}{\omega}\right)^{2}
$$

By formula (7) shows that, when the motor voltage to the inverter can output voltage limit, if it continues to accelerate, which is required to enter the field of weak magnetic control, to increase $\boldsymbol{i}_{\boldsymbol{d}}$ while reducing $\boldsymbol{i}_{q}$ to maintain the output voltage balancing effect.

It can get the current limit circle and voltage limit ellipse by formula (4) and formula (7). As shown in Figure 1, curve OA represents the maximum torque/current control trajectory, When the access to the weak magnetic control, the running track of the stator current is along the curve $\mathrm{AB}$ from point $\mathrm{A}$ to point $\mathrm{B}$, in order to achieve the purpose of weakening.

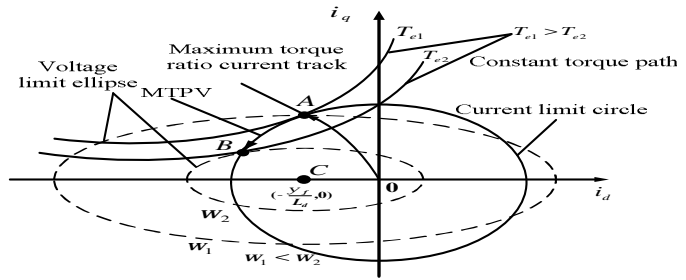

Fig. 1 stator current vector trajectory

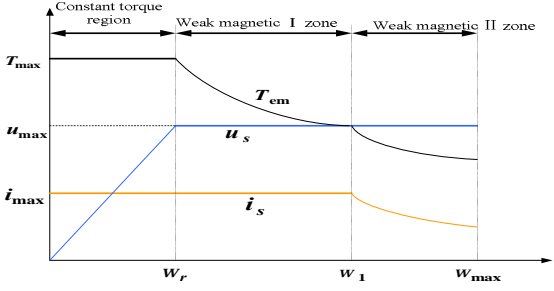

Fig. 2 Weak magnetic control working area

\section{Weak magnetic control region}

IPMSM for the weak magnetic control, working area division of current track and the changes of voltage, current and torque in the area as shown in figure 2, Weak magnetic II region can be considered to be the optimal control area marked in Figure 2, in this area, the maximum power can be achieved when the parameters of the motor meet the specific requirements.

In the constant torque region shown in Figure 2, the output torque is constant, and can be the 
maximum value. The counter electromotive force of the motor is proportional to its rotational speed, and it becomes an upward trend, so the output power is increased continuously.

The output voltage of the inverter reaches the maximum value after the point $A$, and the speed is up to the rated value, which has been shown in Figure 1, in this operation mechanism, due to the limit of the current limit cycle, the speed can't continue to rise along the curve. If the motor speed continues to increase, then enter the weak magnetic control area as shown in figure 2.

In the field of weak magnetic I, the motor speed and torque are inversely proportional to the output power is constant. If higher speed is needed, the control needs to enter the area of the weak magnetic region II, and the motor runs along the MTPV curve.

The equation can be expressed as:

$$
\frac{\partial T_{\mathrm{e}}}{\partial i_{d}} \frac{\partial U_{s}}{\partial i_{q}}-\frac{\partial T_{\mathrm{e}}}{\partial i_{q}} \frac{\partial U_{s}}{\partial i_{d}}=\mathbf{0}
$$

The direction of MTPV is the tangent direction of the trajectory, by the formula (8) can get the following equation:

$$
\begin{gathered}
\left(\begin{array}{l}
W_{d} \\
W_{q}
\end{array}\right)=\left(\begin{array}{c}
-2\left(L_{d}-L_{q}\right) L_{q}^{2} i_{q} \\
-2\left(L_{d}-L_{q}\right) L_{q} i_{q}-2\left(L_{d}-L_{q}\right) L_{d} \Psi_{f}
\end{array}\right) \\
W=\sqrt{W_{d}^{2}+W_{q}^{2}}
\end{gathered}
$$

According to the $\Delta \mathrm{U}$ value, the current reference can be revised as follows:

$$
\begin{aligned}
& \mathrm{N}_{\mathrm{d}}=\left\{\begin{array}{lll}
\Delta \mathrm{UT}_{\mathrm{d}} / \mathrm{T}, & \text { Weak } & \text { magnetic region } I \\
\Delta \mathrm{UW}_{\mathrm{d}} / \mathrm{W}, & \text { Weak } & \text { magnetic region } I I
\end{array}\right. \\
& \mathrm{N}_{\mathrm{q}}=\left\{\begin{array}{lll}
\Delta U \mathrm{UT}_{\mathrm{q}} / \mathrm{T}, & \text { Weak magnetic region } I \\
\Delta U \mathrm{WW}_{\mathrm{q}} / \mathrm{W}, & \text { Weak magnetic region } I I
\end{array}\right.
\end{aligned}
$$

In order to make the output voltage does not exceed the maximum voltage of the inverter, the input current of the current regulator can be expressed as follows:

$$
\begin{gathered}
i_{d}^{*}=i_{d}+i_{d, m} \\
i_{q}^{*}=i_{q}+i_{q, m}
\end{gathered}
$$

In the formula: $\boldsymbol{i}_{\boldsymbol{d}}$ and $\boldsymbol{i}_{q}$ are the reference current generated by the MTPA module; $\boldsymbol{i}_{\boldsymbol{d}, \boldsymbol{m}}$ and $\boldsymbol{i}_{q, \boldsymbol{m}}$ are the value of the current reference correction value after PI adjustment; $\boldsymbol{i}_{\boldsymbol{d}}^{*}$ and $\boldsymbol{i}_{q}^{*}$ are corrected current reference values.

\section{Weak magnetic control strategy}

The electric vehicle electric drive system can be output at constant torque at low speed, in order to adapt to the fast starting, climbing, acceleration and other requirements; at high speed to achieve constant power flux weakening control, to meet the requirements of electric vehicle cruise speed, passing, and so on. Considering the characteristics of electric drive system and the special requirements of the design of vehicle IPMSM control system, when the control system of IPMSM is designed, the weak magnetic control strategy is adopted, in order to widen the speed range of the motor, to achieve high-speed constant power operation.

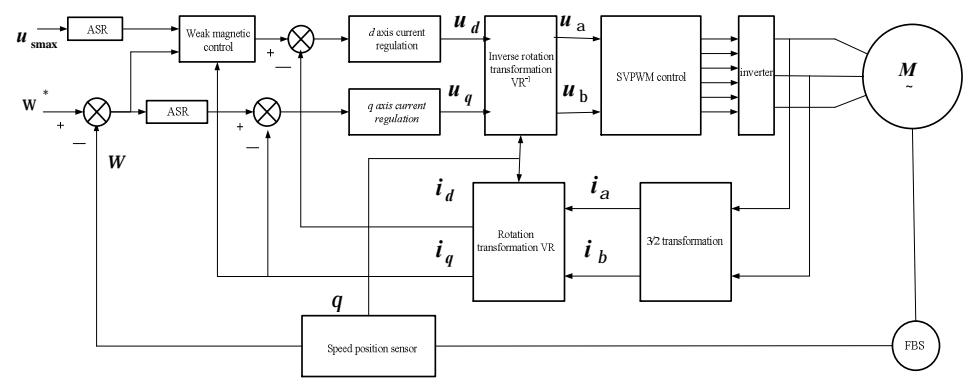

Fig. 3 the overall block diagram of the IPMSM weak magnetic control strategy

Figure 3 is the IPMSM weak magnetic control strategy of the overall block diagram, mainly including: weak magnetic control module, IPMSM motor module, SVPWM generation module, coordinate transformation module, inverter module, speed and current control module and so on. 


\section{System simulation and result analysis}

According to the weak magnetic control principle of permanent magnet synchronous motor, the overall structure diagram of the IPMSM weak magnetic control strategy is given in Figure 3, and the simulation model of IPMSM weak magnetic control system is built in MATLAB by using the above mentioned weak magnetic control strategy, as shown in figure 4 . The parameters of the motor module: stator resistance $\boldsymbol{R}_{s}=0.86 \Omega$, direct axis inductance $\boldsymbol{L}_{\boldsymbol{d}}=0.0055 \mathrm{H}$, cross axis inductance $\boldsymbol{L}_{\boldsymbol{q}}=0.0113 \mathrm{H}$, permanent magnet flux linkage $\psi_{\mathrm{f}}=0.205 \mathrm{~Wb}$, pole pair $\mathrm{p}=4$, moment of inertia $\mathrm{J}=5.345 \times 10^{-3} \mathrm{~kg} \bullet \mathrm{m}^{2}$, viscous friction coefficient $\mathrm{F}=1 \times 10^{-3} \mathrm{~N} \bullet \mathrm{m} \bullet \mathrm{s}$. DC bus voltage $\boldsymbol{U}_{\mathrm{dc}}=537 \mathrm{~V}$, rated speed $\mathrm{N}=3000 \mathrm{r} / \mathrm{min}$.

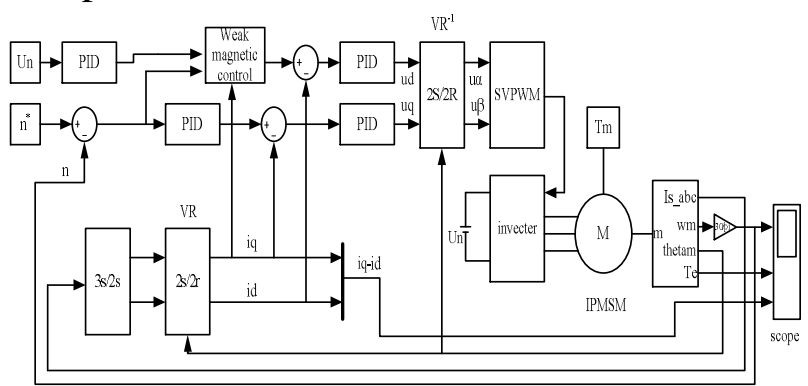

Fig. 4 simulation model of IPMSM weak magnetic control system

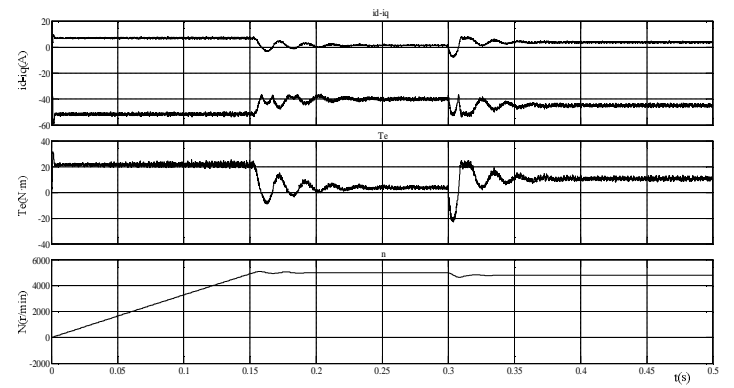

Fig. 5 simulation waveforms of id, iq, torque and speed

When the given speed is $\mathrm{n}=5000 \mathrm{r} / \mathrm{min}$, the IPMSM limiting load system simulation waveform of weak magnetic speed as shown in Figure 5, in the figure, 0 to $0.3 \mathrm{~s}$ of the time period, the load torque is $T_{L}=3 \mathrm{~N} \cdot \mathrm{m} ; 0.3 \mathrm{~s}$ to $0.5 \mathrm{~s}$ of the time period, the load torque is $T_{L}=10 \mathrm{~N} \cdot \mathrm{m}$ Starting torque is $20 \mathrm{~N} \cdot \mathrm{m}$, from Figure 5 shows that ,before $0.15 \mathrm{~s}$, IPMSM system speed up to $5000 \mathrm{r} / \mathrm{min}$, in this period of time, the AC, straight axis current is $i_{d}=-50 \mathrm{~A}, i_{q}=7 \mathrm{~A}$; from $0.15 \mathrm{~s}$ to $0.2 \mathrm{~s}$ is the motor speed regulation time, the electromagnetic torque and load torque are balanced, in this period of time , $i_{q}=1 \mathrm{~A}, i_{d}=-40 \mathrm{~A} ; 0.3 \mathrm{~s}$ later, when $T_{L}=10 \mathrm{~N} \cdot \mathrm{m}$, the electromagnetic torque and load torque are balanced, at this time, $i_{d}=-45 \mathrm{~A}, i_{q}=4 \mathrm{~A}$, speed down to $4800 \mathrm{r} / \mathrm{min}$.Above speed adjustment process, it is proved that the constant power weak magnetic speed control strategy is effective.

\section{Conclusion}

In order to study the IPMSM wide speed control of the vehicle, the basic principle of the weak magnetic expansion is analyzed, and the simulation of the system is made, simulation results show that, after the constant power and weak magnetic speed regulation, the speed of the whole system is raised from the rated speed $3000 \mathrm{r} / \mathrm{min}$ to the speed $5000 \mathrm{r} / \mathrm{min}$, fully proved that the improved speed range, which lays a theoretical foundation for the practical application of high speed cruise and speed of motor vehicle, and provides a good reference value for the research of high performance vehicle motor controller.

\section{Reference:}

[1] Seung-ki Sul, Zhang Yongchang,Li Zhengxi,et al.Motor drive system control[M].Beijing: Machinery Industry Press,2013,10.

[2]Sheng Yifa,Yu Shouyi. Field Weakening Operation Control Strategies of Permanent Magnet Synchronous Motor for Railway Vehicles[J]. Proceedings of the CSEE, 2010,(30):74-79.

[3]Luo Derong,Ceng Zhibo,Huang Shoudao,et al. A Leading Ang le Field Weakening Control Method for Permanent Magnet Synch ronous Moto r on Electric Vehicles[J]. Jour nal of H unan Univer sity. 2011,38(3):1-5.

[4]Tang Chaohui,Ding Qiang,Yu Shouyi,et al. Research of flux weakening strategy of interior permanent magnet synchronous motor[J]. ELECTRIC MACHINES AND CONTROL. 2010, 14 (5):68-72. 
[5]Yu Jiabin,Qin Xiaofei,Zheng Jun,et al. Improved leading angle flux weakening control algorithm[J]. ELECTRIC MACHINES AND CONTROL. 2012, 16(3):101-106. 